\title{
ON A CERTAIN TRANSITIVITY OF THE GRADED RING ASSOCIATED WITH AN IDEAL
}

\author{
NGO VIẸT TRUNG
}

\begin{abstract}
A simple but useful result will be given concerning a certain transitivity of the property that the graded ring associated with an ideal is a domain. As a consequence, we compute the graded rings associated with the defining prime ideals of certain determinantal varieties or of their projections from infinity to a hyperplane and get two new classes of primes having the equality of ordinary and symbolic powers in polynomial rings over a field.
\end{abstract}

1. Introduction. To determine whether ordinary and symbolic powers of a given prime ideal $\mathfrak{p}$ in a Noetherian ring $R$ are equal is often difficult and only a few classes of primes having this equality in polynomial rings over a field are known (see $[1,4,6-10])$. This equality can be deduced from the property of being a domain of the associated graded ring $\operatorname{gr}_{\mathfrak{p}}(R)$, and, if $\operatorname{gr}_{\mathfrak{p} R_{\mathfrak{p}}}\left(R_{\mathfrak{p}}\right)$ is assumed to be a domain, the converse of this deduction also holds [4]. For this reason, in order to find new classes of such primes we will study a sort of transitivity of the above property, which may be formulated as follows: Let $R \rightarrow S$ be a surjective homomorphism of Noetherian rings whose kernel is a complete intersection ideal in $R$. Let $\mathfrak{p}$ be an ideal in $R$ such that $\operatorname{gr}_{\mathfrak{p}}(R)$ is a domain. When is $\operatorname{gr}_{\mathfrak{p} S}(S)$ a domain? (See [1,7] for another sort of transitivity.) A simple but useful result concerning this question will be given (Theorem 1), which allows us to compute the graded rings associated with the defining prime ideals of certain determinantal varieties or of their projections from infinity to a hyperplane and to show that some of them are Cohen-Macaulay domains (\$3). As a consequence, we get two new classes of primes having the equality of ordinary and symbolic powers in polynomial rings over a field. Note that certain Veronesean ideals and the prime ideal of Macaulay are also to be found among such primes.

2. The main result. Let $R$ be a commutative Noetherian ring with identity and $\mathfrak{p}$ an ideal in $R$. We denote by $\operatorname{gr}_{\mathfrak{p}}(R)$ the graded ring $\bigoplus_{n=0}^{\infty} \mathfrak{p}^{n} / \mathfrak{p}^{n+1}$. Given an element $a \notin \mathfrak{p}$ in $R$, we denote by $a^{*}$ the image of $a$ in $R / \mathfrak{p}$ and $a^{*}$ will always be considered as an element of $\operatorname{gr}_{\mathfrak{p}}(R)$. If $\mathfrak{m} \supseteq \mathfrak{p}$ is an ideal in $R$, then we denote by $V(\mathfrak{m})$ the set of all primes in $R$ which contain $\mathfrak{m}$ and by $\mathfrak{m}^{*}$ the ideal in $\operatorname{gr}_{\mathfrak{p}}(R)$ generated by all elements $a^{*}$ with $a \in \mathfrak{m} \backslash \mathfrak{p}$.

Received by the editors March 23, 1979 and, in revised form, September 10, 1980.

AMS (MOS) subject classifications (1970). Primary 13A15, 13H10; Secondary 13C15, 14M05.

Key words and phrases. Ordinary and symbolic powers, graded ring associated with an ideal, CohenMacaulay ring, determinantal ideal, Veronesean variety, projection from infinity to a hyperplane. 
Our main result on the transitivity is the following:

THEOREM 1. Let $\operatorname{gr}_{\mathfrak{p}}(R)$ be a domain and $a_{1}, \ldots, a_{n}$ be a family of elements in $R$ such that $\mathrm{ht}\left(\mathfrak{p}, a_{1}, \ldots, a_{n}\right) / \mathfrak{p}=n$. Let $S$ denote the factor ring $R /\left(a_{1}, \ldots, a_{n}\right)$. Then $\operatorname{gr}_{\mathfrak{p} S}(S) \cong \mathrm{gr}_{\mathfrak{p}}(R) /\left(a_{1}^{*}, \ldots, a_{n}^{*}\right)$ and it is a domain if there exists an ideal $\mathfrak{m} \supset$ ( $\left.\mathfrak{p}, a_{1}, \ldots, a_{n}\right)$ in $R$ such that the following conditions are satisfied:

(i) $\left(\mathfrak{p}, a_{1}, \ldots, a_{i}\right)$ is a locally complete intersection prime in $\operatorname{Spec}(R) \backslash V(\mathrm{~m})$ for all $i=1, \ldots, n$;

(ii) $\left(\mathfrak{p}, a_{1}, \ldots, a_{i}\right)$ has only one minimal associated prime which does not contain $\mathrm{m}$, for all $i=1, \ldots, n$;

(iii) $\operatorname{grade} \mathrm{m}^{*}>n$.

Proof. We prove the theorem by induction on $n$.

Case $n=1$ : Since $\operatorname{gr}_{\mathfrak{p}}(R)$ is a domain and $a_{1} \notin \mathfrak{p}$ (because $\left.\mathrm{ht}\left(\mathfrak{p}, a_{1}\right) / \mathfrak{p}=1\right), a_{1}^{*}$ is not a zero divisor in $\operatorname{gr}_{\mathfrak{p}}(R)$, or, $\mathfrak{p}^{n}: a_{1}=\mathfrak{p}^{n}$ for all $n \geqslant 0$. Thus

$$
\begin{aligned}
\operatorname{gr}_{\left(\mathfrak{p}, a_{1}\right) /\left(a_{1}\right)}\left(R /\left(a_{1}\right)\right) & =\bigoplus_{n=0}^{\infty}\left(\mathfrak{p}^{n}, a_{1}\right) /\left(\mathfrak{p}^{n+1}, a_{1}\right) \\
& \cong \bigoplus_{n=0}^{\infty} \mathfrak{p}^{n} /\left(\mathfrak{p}^{n+1}, a_{1}\left(\mathfrak{p}^{n}: a_{1}\right)\right) \\
& =\bigoplus_{n=0}^{\infty} \mathfrak{p}^{n} /\left(\mathfrak{p}^{n+1}, a_{1} \mathfrak{p}^{n}\right)=\operatorname{gr}_{\mathfrak{p}}(R) /\left(a_{1}^{*}\right) .
\end{aligned}
$$

It remains to show that $\left(a_{1}^{*}\right)$ is a prime ideal. Let $Q$ be an arbitrary associated prime of $\left(a_{1}^{*}\right)$. Then we must show that $\left(a_{1}^{*}\right)=Q$. First, $Q \nsubseteq \mathrm{m}^{*}$ because of (iii). Let $q$ denote the inverse image in $R$ of the restriction of $Q$ in $R / \mathfrak{p}$. Then $q$ is a prime ideal and $q \nsupseteq \mathrm{m}$. It follows from (i) that $\left(\mathfrak{p}, a_{1}\right) R_{\mathrm{q}}$ is a complete intersection prime. Since $\operatorname{ht}\left(\mathfrak{p}, a_{1}\right) / \mathfrak{p}=1, \mathfrak{p} R_{\mathfrak{q}}$ is a complete intersection prime too. Hence, $\operatorname{gr}_{\mathfrak{p} R_{\mathfrak{q}}}\left(R_{\mathfrak{a}}\right)$ is isomorphic to a polynomial ring over $R_{\mathrm{q}} / \mathfrak{p} R_{\mathrm{q}}$ and $a_{1}$ generates a prime ideal in $\operatorname{gr}_{\mathrm{p} R_{\mathrm{q}}}\left(R_{\mathrm{q}}\right)$. Note that $\left[\operatorname{gr}_{\mathrm{p}}(R)\right]_{Q}$ is a localization of $\mathrm{gr}_{\mathrm{p} R_{\mathrm{q}}}\left(R_{\mathrm{q}}\right)$. So we have the following immediate facts: the $Q$-primary component of $\left(a_{1}^{*}\right)$ is $Q$ itself; $Q$ is the only associated prime of $\left(a_{1}^{*}\right)$ whose restriction in $R / \mathfrak{p}$ is $q / \mathfrak{p}$; and $q$ is a minimal associated prime of $\left(\mathfrak{p}, a_{1}\right)$. But by (ii) there exists only one such prime $a$. Hence, summarizing all these facts, we get $\left(a_{1}^{*}\right)=Q$, as required.

Case $n>1$ : Let $\bar{R}=R /\left(a_{1}\right)$. Noting that $h t\left(\mathfrak{p}, a_{1}\right) / \mathfrak{p}=1$ because $\operatorname{ht}\left(\mathfrak{p}, a_{1}, \ldots, a_{n}\right) / \mathfrak{p}=n$, we can first show as above that

$$
\mathrm{gr}_{\mathfrak{p}}-(\bar{R}) \cong \mathrm{gr}_{\mathfrak{p}}(R) /\left(a_{1}^{*}\right)
$$

and that $\operatorname{gr}_{\mathfrak{p} R}(\bar{R})$ is a domain. Let $\bar{a}_{2}, \ldots, \bar{a}_{n}$ denote the images of $a_{2}, \ldots, a_{n}$ in $\bar{R}$. Then it is easy to verify from the assumptions that $\operatorname{ht}\left(\mathfrak{p} \bar{R}, \bar{a}_{2}, \ldots, \bar{a}_{n}\right) / \mathfrak{p} \bar{R}=n-1$ and that

(i') $\left(\mathfrak{p} \bar{R}, \bar{a}_{2}, \ldots, \bar{a}_{i}\right)$ is a locally complete intersection prime in $\operatorname{Spec}(\bar{R}) \backslash V(\mathfrak{m} \bar{R})$, for all $i=2, \ldots, n$, and

(ii') $\left(\mathfrak{p} \bar{R}, \bar{a}_{2}, \ldots, \bar{a}_{i}\right)$ has only one minimal associated prime which does not contain $\mathrm{m} \bar{R}$, for all $i=2, \ldots, n$, and

(iii') $\operatorname{grade}(\mathfrak{m} \bar{R})^{*}>n-1$. 
Now we can apply the induction hypothesis to $\bar{R}$ and $S$. As a consequence,

$$
\operatorname{gr}_{\mathfrak{p} S}(S) \cong \operatorname{gr}_{\mathfrak{p}} \bar{R}(\bar{R}) /\left(\bar{a}_{2}^{*}, \ldots, \bar{a}_{n}^{*}\right)
$$

and it is a domain. It remains to show that $\operatorname{gr}_{\mathfrak{p} S}(S) \cong \mathrm{gr}_{\mathrm{p}}(R) /\left(a_{1}^{*}, \ldots, a_{n}^{*}\right)$. But this follows from (1) and (2) immediately. The proof is complete.

REMARK. (1) If we furthermore require in Theorem 1 that $R_{\mathrm{q}} /\left(\mathfrak{p}, a_{1}, \ldots, a_{n}\right)$ be normal for all primes $a \nsupseteq m$ and grade $m^{*}>n+1$, then, using the Serre criterion on normality, we can also show that $\operatorname{gr}_{\mathfrak{p} S}(S)$ is normal.

(2) The condition (iii) of Theorem 1 is satisfied if $_{g_{p}}(R)$ is a Cohen-Macaulay ring and ht $\mathrm{m}^{*}>n$. Thus, it is easily verifiable in practical uses. For, if $R$ is regular and $\operatorname{gr}_{\mathfrak{p}}(R)$ a domain, then the Rees ring $R\left[\mathrm{p} t, t^{-1}\right]$ is a unique factorization domain by [4, Theorem 1]; hence, by the well-known conjecture of Samuel on the CohenMacaulay property of unique factorization domains, $R\left[\mathrm{p} t, t^{-1}\right]$ and therefore $\mathrm{gr}_{\mathrm{p}}(R)$ $\cong R\left[\mathrm{p} t, t^{-1}\right] /\left(t^{-1}\right)$ are possibly Cohen-Macaulay rings in all such cases. Until now we could not find a counterexample for that (see $[4,8,9,10]$ and $\S 3$ below).

Corollary 2. Let $\operatorname{gr}_{\mathfrak{p}}(R)$ be a Cohen-Macaulay domain. Let $\mathfrak{m} \supseteq \mathfrak{p}$ be an ideal in $R$ such that $\mathfrak{p}$ is a locally complete intersection prime in $\operatorname{Spec}(R) \backslash V(\mathfrak{m})$. For all rings $S$ of the form $R /\left(a_{1}, \ldots, a_{n}\right)$, where $a_{1}, \ldots, a_{n}$ is a family of elements in $\mathfrak{m}$ with $n<\mathrm{ht} \mathrm{m}^{*}$ such that $\mathfrak{p} \subset\left(\mathfrak{p}, a_{1}\right) \subset \cdots \subset\left(\mathfrak{p}, a_{1}, \ldots, a_{n}\right)$ is a chain of primes, $\operatorname{gr}_{\mathfrak{p S}}(S)$ $\cong \mathrm{gr}_{\mathfrak{p}}(R) /\left(a_{1}^{*}, \ldots, a_{n}^{*}\right)$ and it is a Cohen-Macaulay domain.

REMARK. Assume that $R$ is a local ring with the maximal ideal $m$. By [5, Remark 4.1] it is easy to verify that ht $\mathrm{m}^{*} \geqslant \mathrm{ht} M-\mathrm{ht} M / \mathrm{m}^{*} \geqslant \mathrm{ht} M-\operatorname{dim} \mathrm{gr}_{\mathfrak{p}}(R) / \mathrm{m}^{*}=$ $\operatorname{dim} R-l(\mathfrak{p})$, where $M$ denotes the only maximal homogeneous ideal of $\operatorname{gr}_{\mathfrak{p}}(R)$ and $l(\mathfrak{p})$ is the analytic spread of $\mathfrak{p}$ in $R$, which is defined to be the dimension of $\bigoplus_{n=0}^{\infty} \mathfrak{p}^{n} / \mathfrak{m p}^{n} \cong \operatorname{gr}_{\mathfrak{p}}(R) / \mathfrak{m}^{*}$. Therefore, from Corollary 2 we can get a result which connects the transitivity of the property of being a Cohen-Macaulay domain from $\operatorname{gr}_{\mathfrak{p}}(R)$ to $\operatorname{gr}_{\mathfrak{p} S}(S)$ with the analytic spread $l(\mathfrak{p})$. Note that in certain situations there is a close connection between $l(\mathfrak{p})$ and the property of being a domain of $\operatorname{gr}_{\mathfrak{p}}(R)$ [3, Proposition 3].

Corollary 3. Let $\operatorname{gr}_{\mathfrak{p}}(R)$ be a domain. Let $\mathfrak{m} \supset \mathfrak{p}$ be an ideal and $a \notin \mathfrak{p}$ an element in $\mathrm{m}$ such that the following conditions are satisfied:

(i) $\mathfrak{p}$ is a locally complete intersection prime in $\operatorname{Spec}(R) \backslash V(\mathfrak{m})$;

(ii) $(\mathfrak{p}, a)$ is a prime ideal which does not contain $\mathfrak{m}$.

Then $\operatorname{gr}_{(\mathfrak{p}, a) /(a)}(R /(a))$ is a domain if and only if grade $\mathrm{m}^{*}>1$.

Proof. We first have $\operatorname{gr}_{(\mathfrak{p}, a) /(a)}(R /(a)) \cong \operatorname{gr}_{\mathfrak{p}}(R) /\left(a^{*}\right)$ as in the proof of Theorem 1 for the case $n=1$. If grade $\mathrm{m}^{*} \leqslant 1$, there exists a prime ideal $Q \supseteq \mathfrak{m}^{*}$ in $\operatorname{gr}_{\mathfrak{p}}(R)$ such that $\operatorname{depth}\left[\operatorname{gr}_{\mathfrak{p}}(R)\right]_{Q} \leqslant 1$. Hence $Q$ must be an associated prime of $\left(a^{*}\right)$ because $a^{*}$ is a non-zero-divisor in $Q$. But, from (i) and (ii) we easily see that $\left(a^{*}\right)$ also has an associated prime which does not contain $\mathrm{m}^{*}$. Therefore $\left(a^{*}\right)$ is not a prime ideal. We have just shown the implication $(\Rightarrow)$ of Corollary $3 ;(\Leftarrow)$ is only a special case of Theorem 1 . 
3. Applications. We start with the ideal $I$ generated by the $2 \times 2$ minors of the matrix

$$
X=\left(\begin{array}{l}
x_{11} \cdots x_{1 r} \\
x_{21} \cdots x_{2 r}
\end{array}\right), \quad r \geqslant 2,
$$

of indeterminates in $k[X]$, where $k$ is an arbitrary field.

It is clear that $I$ generates a complete intersection prime in $k\left[X, x_{i j}^{-1}\right]$ for all $x_{i j} \in X$. Hence $I$ is a locally complete intersection prime in $\operatorname{Spec}(k[X]) \backslash V(X)$. Let $Y=\left\{y_{i j}: 1 \leqslant i<j \leqslant r\right\}$ be a set of indeterminates. Set $y_{i i}=0, y_{j i}=-y_{i j}$, and

$$
\begin{aligned}
a_{i j l} & =x_{1 i} y_{j l}-x_{1 j} y_{i l}+x_{1 l} y_{i j}, \\
b_{i j l} & =x_{2 i} y_{j l}-x_{2 j} y_{i l}+x_{2 l} y_{i j}, \\
c_{i j l m} & =y_{i m} y_{j l}-y_{j m} y_{i l}+y_{l m} y_{i j},
\end{aligned}
$$

for all $i, j, l, m=1, \ldots, r$. Let $J$ denote the ideal in $k[X, Y]$ generated by $I$ and all elements $a_{i j l}, b_{i j l}, c_{i j l m}$. By [8, Applications], $\operatorname{gr}_{I}(k[X]) \cong k[X, Y] / J$ and it is a Cohen-Macaulay domain of dimension $2 r$. Further, $\operatorname{gr}_{I}(k[X]) /(X)^{*}$ is isomorphic to the coordinate ring of the Grassmanian variety $G_{2, r}$ over $k$, which has dimension $2 r-3$; hence

$$
\mathrm{ht}(X)^{*}=\operatorname{dim}_{\mathrm{gr}_{I}}(k[X])-\operatorname{dim} \operatorname{gr}_{I}(k[X]) /(X)^{*}=3 .
$$

Let $a_{1}, \ldots, a_{r-1}$ denote the elements $x_{12}-x_{21}, \ldots, x_{1 r}-x_{2 r-1}$. It is known that $k[X] /\left(I, a_{1}, \ldots, a_{r-1}\right)$ is isomorphic to the coordinate ring of the Veronesean variety $V_{2, r}[2, \S 4]$, which has dimension 3. Thus, $\left(I, a_{1}, \ldots, a_{r-1}\right)$ is a prime ideal with $\operatorname{ht}\left(I, a_{1}, \ldots, a_{r-1}\right) / I=\operatorname{dim} k[X] / I-\operatorname{dim} k[X] /\left(I, a_{1}, \ldots, a_{r-1}\right)=r+2-3=r$ -1 . From this we can then deduce that $\left(I, a_{1}\right),\left(I, a_{1}, a_{2}\right)$, and $\left(I, a_{1}, a_{2}, a_{3}\right)$ are prime ideals. Therefore, applying Corollary 2 and Corollary 3 , we easily get

Proposition 4. Let

$$
\begin{array}{ll}
X_{1}=\left(\begin{array}{lll}
x_{11} \cdots x_{1 r-3} & x_{1} x_{2} x_{3} \\
x_{21} \cdots x_{2 r-3} & x_{2} x_{3} x_{4}
\end{array}\right), & r \geqslant 3, \\
X_{2}=\left(\begin{array}{lll}
x_{11} \cdots x_{1 r-4} & x_{1} x_{2} x_{3} x_{4} \\
x_{21} \cdots x_{2 r-4} & x_{2} x_{3} x_{4} x_{5}
\end{array}\right), & r \geqslant 4,
\end{array}
$$

be matrices of indeterminates. Let $I_{i}, i=1,2$, denote the ideal in $k\left[X_{i}\right]$ generated by the $2 \times 2$ minors of $X_{i}$. Then the following statements hold:

(i) $\operatorname{gr}_{I_{1}}\left(k\left[X_{1}\right]\right) \cong k[X, Y] /\left(J, a_{1}, a_{2}\right)$ and it is a Cohen-Macaulay domain;

(ii) $\operatorname{gr}_{I_{2}}\left(k\left[X_{2}\right]\right) \cong k[X, Y] /\left(J, a_{1}, a_{2}, a_{3}\right)$ and it is a Cohen-Macaulay ring which is not a domain.

ExAmple. Let $\mathfrak{p}$ denote the ideal in $R=k\left[x_{1}, x_{2}, x_{3}, x_{4}\right]$ generated by the following polynomials

$$
p_{1}=x_{1} x_{3}-x_{2}^{2}, \quad p_{2}=x_{1} x_{4}-x_{2} x_{3}, \quad p_{3}=x_{2} x_{4}-x_{3}^{2} .
$$

Then $\mathfrak{p}$ is the defining ideal of the Veronesean variety $V_{2,3}$ in $R$. Let $y_{1}, y_{2}, y_{3}$ be indeterminates over $R$ and set

$$
p_{4}=x_{1} y_{1}-x_{2} y_{2}+x_{3} y_{3}, \quad p_{5}=x_{2} y_{1}-x_{3} y_{2}+x_{4} y_{3} \text {. }
$$


By Proposition 4(i), $\operatorname{gr}_{\mathrm{p}}(R) \cong R\left[y_{1}, y_{2}, y_{3}\right] /\left(p_{1}, \ldots, p_{5}\right)$ and $\left(p_{1}, \ldots, p_{5}\right)$ is a perfect prime ideal. Therefore, $\mathfrak{p}^{(n)}=\mathfrak{p}^{n}$ for all $n \geqslant 0$. In the same way we can also compute the associated graded ring with respect to the defining prime ideal of the Veronesean variety $V_{2,4}$ by using Proposition 4(ii). It is not a domain. Hence, we do not have the equality of ordinary and symbolic powers in this case, which was already known in [7, Example 1]. In [1] it was even shown that the defining prime ideal of $V_{2, r}$ with $r \geqslant 4$ does not have this equality.

In the following we will consider the projection of the variety of the zeros of the ideal of the $2 \times 2$ minors of the matrix

$$
\left(\begin{array}{ccc}
x_{11} \cdots x_{1 r} & x_{1} & u \\
x_{21} \cdots x_{2 r} & u & x_{2}
\end{array}\right)
$$

from infinity to the hyperplane $u=0$.

Let $I^{\prime}$ denote the defining prime ideal of the variety obtained by this projection in $k\left[X^{\prime}\right]=k\left[X, x_{1}, x_{2}\right]$. By [10], $I^{\prime}$ is generated by $I$ and the elements $x_{1} x_{2 i} x_{2 j}-$ $x_{2} x_{1 i} x_{1 j}, i, j=1, \ldots, r$. Let $Z=\left\{z_{i j}: 1 \leqslant i \leqslant j \leqslant r\right\}$ be a set of indeterminates. Set $z_{j i}=z_{i j}$ and

$$
\begin{aligned}
d_{i j l m} & =y_{j l} z_{i m}-y_{j m} z_{i l}+y_{l m} z_{i j}, \\
f_{i j l} & =x_{1 i} z_{j l}-x_{1 j} z_{i l}-x_{1} x_{2 l} y_{i j}, \\
g_{i j l} & =x_{2 i} z_{j l}-x_{2 j} z_{i l}-x_{2} x_{1 l} y_{i j}, \\
h_{i j l m} & =z_{i m} z_{j l}-z_{i l} z_{j m}-x_{1} x_{2} y_{l m} y_{i j}
\end{aligned}
$$

for all $i, j, l, m=1, \ldots, r$. Let $J^{\prime}$ denote the ideal in $k\left[X^{\prime}, Y, Z\right]$ generated by $J$ and all elements $d_{i j l m}, f_{i j l}, g_{i j l}, h_{i j l m}$. By $[10], \operatorname{gr}_{I^{\prime}}\left(k\left[X^{\prime}\right]\right) \cong k\left[X^{\prime}, Y, Z\right] / J^{\prime}$ and it is a Cohen-Macaulay domain.

Let $a_{1}^{\prime}=x_{1}-x_{2 r-1}, a_{2}^{\prime}=x_{2}-x_{1 r}$. It is not hard to see that $\left(I^{\prime}, a_{1}^{\prime}\right) k\left[X^{\prime}, x_{11}^{-1}\right]$ is generated by the elements $a_{1}^{\prime}, x_{1} x_{21}^{2}-x_{2} x_{11}^{2}$, and $x_{11} x_{2 i}-x_{1 i} x_{21}, i=1, \ldots, r$, and that therefore

$$
\begin{aligned}
k\left[X^{\prime}, x_{11}^{-1}\right] /\left(I^{\prime}, a_{1}^{\prime}\right) & \cong k\left[x_{11}, \ldots, x_{1 r}, x_{21}, x_{11}^{-1}\right], \\
k\left[X^{\prime}, x_{11}^{-1}\right] /\left(I^{\prime}, a_{1}^{\prime}, a_{2}^{\prime}\right) & \cong k\left[x_{11}, \ldots, x_{1 r-1}, x_{21}, x_{11}^{-1}\right] .
\end{aligned}
$$

It follows that $\left(I^{\prime}, a_{1}^{\prime}\right) k\left[X^{\prime}, x_{11}^{-1}\right]$ and $\left(I^{\prime}, a_{1}^{\prime}, a_{2}^{\prime}\right) k\left[X^{\prime}, x_{11}^{-1}\right]$ are complete intersection primes and that the elements of the set $X \backslash\left\{x_{11}\right\}$ are not zero-divisors on these ideals. Since the same facts also hold if we replace $x_{11}$ by an arbitrary indeterminate of $X,\left(I^{\prime}, a_{1}^{\prime}\right)$ and $\left(I^{\prime}, a_{1}^{\prime}, a_{2}^{\prime}\right)$ are locally complete intersection primes in $\operatorname{Spec}\left(k\left[X^{\prime}\right]\right) \backslash V\left(X^{\prime}\right)$, and each of these ideals only has one associated prime which does not contain $\left(X^{\prime}\right)=\left(X, a_{1}^{\prime}, a_{2}^{\prime}\right)$.

Next we will show that grade $\left(X^{\prime}\right)^{*} \geqslant 3$. Note that $\operatorname{gr}_{I^{\prime}}\left(k\left[X^{\prime}\right]\right) /\left(X^{\prime}\right)^{*} \cong k[Y, Z] / P$, where $P$ denotes the ideal in $k[Y, Z]$ generated by all elements $c_{i j l m}, d_{i j l m}$, and $z_{i m} z_{j l}-z_{i l} z_{j m}, i, j, l, m=1, \ldots, r$. We have

$$
\begin{aligned}
\operatorname{ht}\left(X^{\prime}\right)^{*} & =\operatorname{dim} \operatorname{gr}_{I^{\prime}}\left(k\left[X^{\prime}\right]\right)-\operatorname{dim} k[Y, Z]+\mathrm{ht} P \\
& =2 r+2-r^{2}+\text { ht } P .
\end{aligned}
$$


To estimate ht $P$ we must consider a large class of ideals. Let $P_{s}, 1 \leqslant s \leqslant r$, denote the ideal in $k[Y, Z]$ generated by $P$ and all elements $y_{i j}$ with $i, j=1, \ldots, s\left(P=P_{1}\right)$. We claim that ht $P_{s} \geqslant r^{2}-2 r+s$. For $r=2$ it is trivial. So, if we denote by $Q_{s}$ the ideal in $k[Y, Z]$ generated by $P_{s}$ and $y_{12}, \ldots, y_{1 r}, z_{11}, \ldots, z_{1 r}$, we may assume, by using the induction hypothesis on $r-1$, that ht $Q_{s} \geqslant\left[(r-1)^{2}-2(r-1)+\right.$ $(s-1)]+2 r-1=r^{2}-2 r+s+1$. Further, it is easily seen by [2, \$4, Corollary] that $P_{r}$ (which is generated by $Y$ and the $2 \times 2$ minors of the symmetric $r \times r$ matrix $\left.\left(z_{i j}\right)\right)$ is a prime ideal of height $r^{2}-r$. Hence, by descending induction we may also assume that $s<r$ and ht $P_{s+1} \geqslant r^{2}-2 r+s+1$. Since $\sqrt{\left(P_{s}, y_{1 s+1}\right)}=\sqrt{P_{s+1} \cap Q_{s}}$ which is easily verified, $\mathrm{ht}\left(P_{s}, y_{1 s+1}\right) \geqslant r^{2}-2 r+s+1$. Hence ht $P_{s} \geqslant r^{2}-2 r+s$, as claimed. (It may even be shown that $P_{s}$ is a perfect prime ideal with ht $P_{s}=r^{2}-$ $2 r+s$ by using the techniques of [10].) As a consequence, $\mathrm{ht}\left(X^{\prime}\right)^{*} \geq 2 r+2-r^{2}+$ $r^{2}-2 r+1=3$. From this it follows that grade $\left(X^{\prime}\right)^{*} \geqslant 3$ because $\operatorname{gr}_{r^{\prime}}\left(k\left[X^{\prime}\right]\right)$ is a Cohen-Macaulay domain.

Now, applying Theorem 1 to the above situation, we get

Proposition 5. Let $I_{3}$ denote the ideal in $k[X]$ generated by $I$ and the elements $x_{1 i} x_{1 j} x_{1 r}-x_{2 i} x_{2 j} x_{2 r-1}, i, j=1, \ldots, r$. Then $\operatorname{gr}_{I_{3}}(k[X]) \cong k\left[X^{\prime}, Y, Z\right] /\left(J^{\prime}, a_{1}^{\prime}, a_{2}^{\prime}\right)$ and it is a Cohen-Macaulay domain. Further, $I_{3}$ is the defining prime ideal of the variety obtained by the projection of the variety of the zeros of the $2 \times 2$ minors of the matrix

$$
\left(\begin{array}{cccc}
x_{11} \cdots & x_{1 r} & x_{2 r-1} & u \\
x_{21} \cdots & x_{2 r} & u & x_{1 r}
\end{array}\right)
$$

from infinity to the hyperplane $u=0$.

Proof. By the above considerations the first statement is immediate. From this statement we see that $I_{3}$ is a prime ideal with ht $I_{3}=r$. Since the defining prime ideal of the projection-variety contains $I_{3}$ and has height $r$, it is just $I_{3}$. We have proved the second statement.

EXAMPLE. Let $\mathfrak{p}$ denote the ideal in $R=k\left[x_{1}, x_{2}, x_{3}, x_{4}\right]$ generated by the following polynomials:

$$
\begin{array}{ll}
q_{1}=x_{3} x_{4}-x_{1} x_{2}, & q_{2}=x_{1}^{2} x_{4}-x_{2}^{2} x_{3}, \\
q_{3}=x_{1}^{3}-x_{2} x_{3}^{2}, & q_{4}=x_{1} x_{4}^{2}-x_{2}^{3} .
\end{array}
$$

Then $\mathfrak{p}$ is the well-known prime ideal of Macaulay, which is the defining prime ideal of a projection of the Veronesean variety $V_{2,4}$. Let $y_{1}, y_{2}, y_{3}, y_{4}$ be indeterminates over $R$ and set

$$
\begin{aligned}
& q_{5}=x_{3} y_{2}-x_{2} y_{3}-x_{1}^{2} y_{1}, \\
& q_{6}=x_{3} y_{3}-x_{2} y_{4}-x_{1} x_{4} y_{1}, \\
& q_{7}=x_{1} y_{2}-x_{4} y_{3}-x_{2} x_{3} y_{1}, \\
& q_{8}=x_{1} y_{3}-x_{4} y_{4}-x_{2}^{2} y_{1}, \\
& q_{9}=y_{3}^{2}-y_{2} y_{4}-x_{1} x_{2} y_{1}^{2} .
\end{aligned}
$$


By Proposition 5, $\operatorname{gr}_{\mathfrak{p}}(R) \cong R\left[y_{1}, y_{2}, y_{3}, y_{4}\right] /\left(q_{1}, \ldots, q_{9}\right)$ and $\left(q_{1}, \ldots, q_{9}\right)$ is a perfect prime ideal. Therefore, $\mathfrak{p}^{(n)}=\mathfrak{p}^{n}$ for all $n \geqslant 0$. These facts were known in [9], where the proof was more complicated. We also point out that the defining prime ideals of all simple projections of the Veronesean variety $V_{2, r}$ with $r \geqslant 7$ do not have the equality of ordinary and symbolic powers. In the case $r=5,6$ there are only few such primes having this equality.

ACKNOWLEgment. The author wishes to express his sincere thanks to the referee for helpful suggestions relating to Theorem 1.

\section{REFERENCES}

1. R. Achilles, P. Schenzel and W. Vogel, Bemerkungen über normale Flachheit und normale Torsionsfreiheit und Anwendungen, Martin-Luther-Universität, Sektion Mathematik, Halle, DDR, 1976 (preprint).

2. J. Barshay, Determinantal varieties, monomial semigroups, and algebras associated with ideals, Proc. Amer. Math. Soc. 40 (1973), 16-22.

3. R. C. Cowsik and M. V. Nori, On the fibres of blowing up, J. Indian Math. Soc. (N.S.) 40 (1976), 217-222.

4. M. Hochster, Criteria for equality of ordinary and symbolic powers of primes, Math. Z. 133 (1973), 53-65.

5. M. Hochster and L. J. Ratliff, Jr., Five theorems on Macaulay rings, Pacific J. Math. 44 (1973), 147-172.

6. L. Robbiano and J. Valla, Primary powers of a prime ideal, Pacific J. Math. 63 (1976), 491-498.

7. On normal flatness and normal torsion-freeness, J. Algebra 43 (1976), 552-560.

8. N. V. Trung, On the symbolic powers of determinantal ideals, J. Algebra 58 (1979), 361-369.

9. __ Der graduierte Ring bezüglich des Primideals von Macaulay, Wiss. Martin-Luther-Univ. Halle-Wittenberg Math.-Natur. Reihe (to appear).

10. Math. Kyoto Univ. 21 (1981).

Institute of Mathematics, Vien ToAn Hoc, Vien KhoA Hoc, Nghĩ Đo, TU' liem, Hanoi, VIETNAM 